\title{
Ultrastrong coupling between THz phonons and photons caused by an enhanced vacuum electric field
}

\author{
Zhenya Zhang ๑, Hideki Hirori $\odot,{ }^{*}$ Fumiya Sekiguchi $\odot$, Ai Shimazaki $\odot$, Yasuko Iwasaki $\odot$, Tomoya Nakamura $\odot$, \\ Atsushi Wakamiya $\bullet$, and Yoshihiko Kanemitsu ${ }^{\dagger}{ }^{\dagger}$ \\ Institute for Chemical Research, Kyoto University, Uji, Kyoto 611-0011, Japan
}

(Received 14 May 2021; accepted 8 July 2021; published 27 July 2021)

\begin{abstract}
Ultrastrong coupling (USC) between phonons and vacuum photons can result in fascinating quantum phenomena, though it is difficult to achieve due to the small dipole moments of phonons in solids. Here, we investigate the vacuum Rabi splitting by coupling phonons in perovskite $\mathrm{CH}_{3} \mathrm{NH}_{3} \mathrm{PbI}_{3}$ films with photons in split ring resonators. As the gap size of the resonator decreases, the coupling strength $\eta$ increases due to the enhanced vacuum field in the gap, reaching the USC regime $(\eta \sim 0.24)$ at a gap size of $100 \mathrm{~nm}$. Our results show that nanoresonators are an excellent platform for studies of vacuum-dressed phonon properties.
\end{abstract}

DOI: 10.1103/PhysRevResearch.3.L032021

Electromagnetic fields are subject to quantum fluctuations; even if the mean value of the electromagnetic field in vacuum is zero, a fluctuation of the field still exists [1-3]. These socalled vacuum fluctuations characterize the ground state of a system. Vacuum fluctuations can lead to a number of observable phenomena, such as spontaneous photon emission [4], the Lamb shift [5,6], and the Casimir force [7,8]. Recently, ultrastrong coupling (USC) between matter and the photons of the vacuum field in a cavity has attracted much attention because it provides new possibilities for studies of nonlinear quantum optics, such as the extraction of virtual photons from the ground state [9-11], and for applications such as quantum information processing and ultrafast devices [12-14].

In materials science, USC is considered useful because coupling of vacuum photons with vibrational modes of molecules or phonons in solids enables us to modify chemical reactions $[15,16]$, and it has been proposed as an alternative method to induce phase transitions in solids $[17,18]$. The normalized coupling strength between matter and cavity photons is defined by $\eta \equiv \Omega_{\mathrm{R}} /\left(2 v_{\mathrm{p}}\right)$, where $\Omega_{\mathrm{R}}$ is the vacuum Rabi frequency and $v_{\mathrm{p}}$ is the photon frequency. USC refers to the coupling regime where $\Omega_{\mathrm{R}}$, which corresponds to the rate of the energy exchange between photons and matter, approaches $v_{\mathrm{p}}(\eta>0.1)$ [19]. Although USC has been achieved in many electronic systems [20-23], it remains difficult to achieve in phononic systems due to the smaller dipole moments of vibrational modes in solids (compared to electronic excitations) [24-27]. Because a stronger coupling is expected to induce more dramatic changes in matter, it is necessary to

\footnotetext{
*hirori@scl.kyoto-u.ac.jp

${ }^{\dagger}$ kanemitu@scl.kyoto-u.ac.jp

Published by the American Physical Society under the terms of the Creative Commons Attribution 4.0 International license. Further distribution of this work must maintain attribution to the author(s) and the published article's title, journal citation, and DOI.
}

understand how the coupling strength between cavity photons and phonons can be enhanced. While the coupling strength in a cavity depends on the vacuum field amplitude, the dipole moment, and the number of dipoles (or oscillators) [4,28], previous studies mainly focused on the role of dipoles [25,29]. Therefore, it has remained unclear if the effect of vacuumfield enhancement can be utilized to realize USC between phonons and photons.

In this work, we studied the coupling strength of phonons of a perovskite $\mathrm{CH}_{3} \mathrm{NH}_{3} \mathrm{PbI}_{3}\left(\mathrm{MAPbI}_{3}\right)$ thin film with photons in a split ring resonator (SRR), which strongly enhances the electric field in its gap at terahertz $(\mathrm{THz})$ frequencies. Because the resonance frequency and the electric field enhancement in an SRR can be tuned by simply varying its dimensions, the SRR design is useful in clarifying the relation between the vacuum field and $\eta$. The metal-halide perovskite $\mathrm{MAPbI}_{3}$ has transverse-optical (TO) phonon modes that lie in the $\mathrm{THz}$ frequency region. These phonons are considered to be an important ingredient in the superior optoelectronic performance of the halide perovskites [30,31]. We used $\mathrm{THz}$ time-domain spectroscopy (THz-TDS) to evaluate the vacuum Rabi splitting of the photon-phonon coupled system from the transmission spectra of our samples. We found that $\eta$ increases as the SRR gap becomes narrower, and that the $\eta$ for a gap size of $100 \mathrm{~nm}$ is well in the USC regime $(\eta \sim 0.24)$. Our analysis of the gap-size dependence shows that the vacuum field that occurs by narrowing the gap can more than compensate for the simultaneous reduction in the contribution of the oscillators inside the cavity, thus leading to a net increase of $\eta$ in SRRs with narrower gaps.

We fabricated 21 different arrays of gold SRRs on $z$-cut quartz substrates by using electron beam lithography. Each fabricated array contained many SRRs with the same design and occupied an area of about $2 \mathrm{~mm} \times 2 \mathrm{~mm}$. A thin film of $\mathrm{MAPbI}_{3}$ with a thickness of $t_{\mathrm{MA}}=100 \mathrm{~nm}$ was spin coated over the fabricated SRRs [32]. As shown in Fig. 1(a), the SRR geometry is characterized by the square size $L$ (we used the range $L=16-28 \mu \mathrm{m})$, the metal width $W=3 \mu \mathrm{m}$, and the 


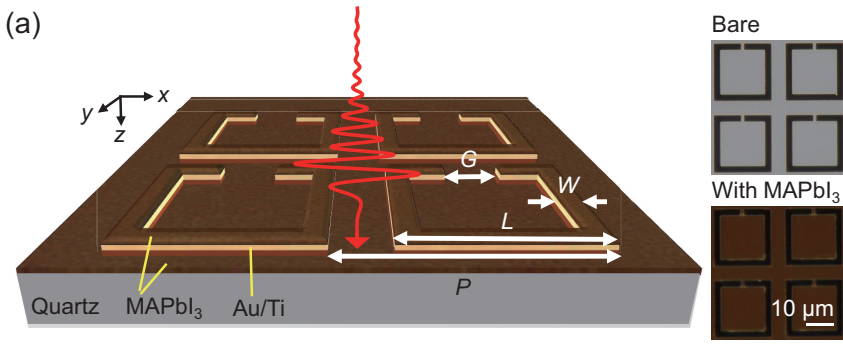

(b)

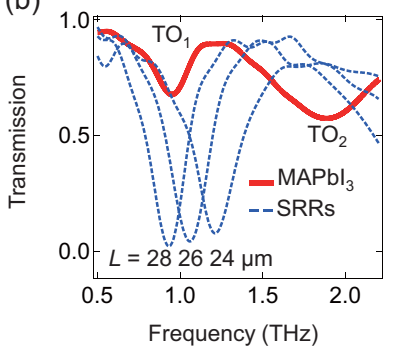

(c)

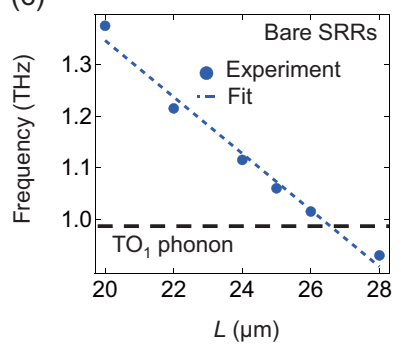

FIG. 1. (a) Left panel: Illustration of SRRs covered with an $\mathrm{MAPbI}_{3}$ thin film. The normally incident $\mathrm{THz}$ pulse is linearly polarized along the $x$ axis. Right panel: Optical microscope images of SRRs with and without $\mathrm{MAPbI}_{3}$. (b) Transmission spectra of an 850-nm-thick $\mathrm{MAPbI}_{3}$ film (red solid curve) and bare SRRs with $G=1 \mu \mathrm{m}$ (blue dashed curves). (c) Resonance frequency of the 1- $\mu$ m-gap bare SRRs as a function of $L$ (blue circles). The blue dashed line is the fit to a linear function. The $\mathrm{TO}_{1}$ phonon frequency of $\mathrm{MAPbI}_{3}$ is indicated by the black dashed line.

gap size $G(=0.1,0.2,0.5$, and $1 \mu \mathrm{m})$. For each value of $G$, we prepared several samples with different values of $L$. The separation between the SRRs was $6 \mu \mathrm{m}$; thus, the period $P=L+6 \mu \mathrm{m}$.

To study the vacuum Rabi splitting, transmission spectra were measured by THz-TDS [32,36]. The incident THz pulse was polarized parallel to the $x$ axis shown in Fig. 1(a). The blue dashed curves in Fig. 1(b) represent the transmission spectra of three bare SRRs with $G=1 \mu \mathrm{m}$. The absorption peak at around $1 \mathrm{THz}$ for each SRR array is attributed to the LC resonance of the SRR [37,38], and it shifts to higher frequencies as $L$ is decreased. At the $L C$ resonance, the electric field is tightly confined and enhanced in the gap. The frequency of the LC mode, which is the cavity resonance frequency $v_{\mathrm{p}}$, can be described by $v_{\mathrm{p}} \approx 1 /\left(2 \pi \sqrt{L_{\mathrm{c}} C}\right)$ [39], where the inductance $L_{\mathrm{c}}$ and the capacitance $C$ are determined by the SRR dimension and the refractive index of the environment. The transmission spectrum of an 850-nm-thick $\mathrm{MAPbI}_{3}$ film without SRRs is shown by the red solid curve in Fig. 1(b). The absorption peaks at 0.95 and $1.86 \mathrm{THz}$ are attributed to the TO phonons of $\mathrm{MAPbI}_{3}$ (hereafter referred to as the $\mathrm{TO}_{1}$ and $\mathrm{TO}_{2}$ phonons, respectively), and they are related to the $\mathrm{Pb}-\mathrm{I}-\mathrm{Pb}$ rocking and $\mathrm{Pb}-\mathrm{I}$ stretching vibrations, respectively [40-42]. The value of $L_{\mathrm{c}}$ can be modified by changing the size $L$ as shown in Fig. 1(c), and thus it is possible to tune $v_{\mathrm{p}}$ over a range that covers the $\mathrm{TO}_{1}$ phonon frequency. When an SRR array is coated with a thin film of MAPbI ${ }_{3}, v_{\mathrm{p}}$ shifts to lower frequencies because of the change in the refractive index around the cavity. Thus, the investigated range $L=16-28 \mu \mathrm{m}$ should cover the phonon line well. (a)

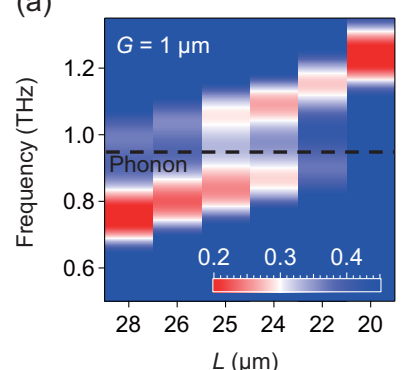

(b)

(c)

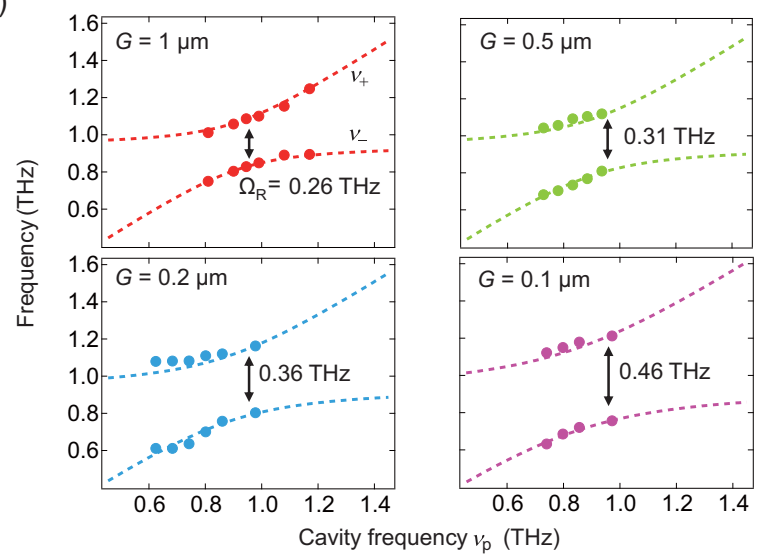

FIG. 2. (a) Two-dimensional map of the transmission spectra for the samples with a gap size of $1 \mu \mathrm{m}$. The position of the $\mathrm{TO}_{1}$ phonon is indicated by the black dashed line. (b) Transmission spectra that correspond to the on-resonance case. The polariton peaks are indicated by triangles. (c) Experimental polariton peak positions (circles) and the theoretical polariton dispersion predicted by the two-oscillator model (dashed curves) for different gap sizes as a function of the cavity resonance frequency. The $x$-axis positions of the black arrows are about $0.95 \mathrm{THz}$.

In the following, we show the transmission spectra of the SRR samples coated with $\mathrm{MAPbI}_{3}$. The color map in Fig. 2(a) summarizes the transmission spectra of the six samples with $G=1 \mu \mathrm{m}$. The black dashed line indicates the $\mathrm{TO}_{1}$ phonon frequency $v_{\mathrm{TO}_{1}}=0.95 \mathrm{THz}$. When $v_{\mathrm{p}}$ is tuned to $\approx v_{\mathrm{TO}_{1}}$ by changing $L$, the absorption peak of the $\mathrm{LC}$ resonance splits. This split into two peaks indicates the generation of photon-phonon polaritons. Hereafter, the two absorption peaks are referred to as the upper $\left(v_{+}\right)$and lower $\left(v_{-}\right)$polariton peaks. We measured all 21 samples to clarify the gap-size dependence of the splitting [32]. The result is summarized in Fig. 2(b). Here, for each of the four investigated gap sizes, we chose the sample whose transmission spectrum fulfills the condition $v_{\mathrm{p}} \approx v_{\mathrm{TO}_{1}}$ best (e.g., for $G=1 \mu \mathrm{m}$, we chose the sample with $L=24 \mu \mathrm{m}$ ), and we showed its spectrum. Figure 2(b) clearly shows that the split of the peaks becomes larger as the gap becomes narrower. This indicates that the coupling between the cavity photons and the $\mathrm{TO}_{1}$ phonons becomes stronger for smaller values of $G$, as explained below. For the smaller gap sizes $(G=0.1$ and $0.2 \mu \mathrm{m})$, the polariton absorption is relatively weak because of the much smaller volume of coupled $\mathrm{MAPbI}_{3}$, and thus the uncoupled phonon absorption can be observed between them [32] 
The anticrossing behavior of the polaritons shown in Fig. 2(a) is a characteristic feature of (ultra)strong coupling $[25,27]$. To evaluate the coupling strength, we extracted the Rabi frequency $\Omega_{\mathrm{R}}$ for each gap size from the measured polariton frequencies. This analysis is based on the eigenvalues of the full Hopfield Hamiltonian of two coupled oscillators [32]. In the on-resonance case where $v_{\mathrm{p}}=v_{\mathrm{TO}_{1}}$, the polariton peak positions $v_{ \pm}$are

$$
v_{ \pm}=\sqrt{v_{\mathrm{TO}_{1}}^{2}+\frac{\Omega_{\mathrm{R}}^{2}}{4}} \pm \frac{\Omega_{\mathrm{R}}}{2} .
$$

From Eq. (1), we obtain the following two equations:

$$
\begin{gathered}
\frac{v_{+}+v_{-}}{2}=\sqrt{v_{\mathrm{TO}_{1}}^{2}+\frac{\Omega_{\mathrm{R}}^{2}}{4},} \\
v_{+}-v_{-}=\Omega_{\mathrm{R}} .
\end{gathered}
$$

Equation (3) shows that, in the on-resonance case, a stronger coupling results in a larger splitting. By substituting $v_{+}-v_{-}$for $\Omega_{\mathrm{R}}$ in Eq. (2), we find the following condition for the on-resonance case:

$$
\frac{v_{+}+v_{-}}{2}=\sqrt{v_{\mathrm{TO}_{1}}^{2}+\frac{\left(v_{+}-v_{-}\right)^{2}}{4}} .
$$

The sample corresponding to the on-resonance case for a certain gap size can thus be identified by finding the pair of upper and lower polariton frequencies that fulfills the condition given in Eq. (4). Then, by plugging the appropriate pair into Eq. (3), we obtained $\Omega_{R}=0.26,0.31,0.36$, and $0.46 \mathrm{THz}$ for the 1-, 0.5-, 0.2-, and $0.1-\mu \mathrm{m}$-gap samples, respectively. The experimentally obtained peak positions $v_{+}$ and $\nu_{-}$are shown as circles in Fig. 2(c). The dashed curves in Fig. 2(c) describe the anticrossing behavior of $v_{ \pm}$for each gap size calculated by Eq. (S1) in the Supplemental Material [32]. To compare the experimental data (which are a function of $L$ ) with these curves, we estimated $v_{\mathrm{p}}$ from $L$ as explained in the Supplemental Material [32]. As shown in Fig. 2(c), the experimentally obtained anticrossing behavior follows the theoretical polariton dispersion. The four polariton-dispersion graphs clearly visualize that $\Omega_{R}$ becomes larger as the gap becomes narrower. The normalized coupling strength can be calculated using $\eta \equiv \Omega_{\mathrm{R}} /\left(2 v_{\mathrm{p}}\right)$, and we obtained $\eta=0.24$ for the $0.1-\mu \mathrm{m}$-gap SRR. This result confirms that the photonphonon coupling in the gap of this SRR lies well in the USC regime $(\eta>0.1)$.

The Rabi frequency of a coupled system can be expressed in terms of the dipole moment $d$ (e.g., that of the $\mathrm{TO}_{1}$ phonon), the density of the oscillators $N$ (here, oscillators refers to the $\mathrm{Pb}-\mathrm{I}-\mathrm{Pb}$ bonds), the corresponding volume $V_{0}$ of the material inside the coupling region (e.g., the gap of SRR), and the vacuum electric field amplitude $\left|E_{\text {vac }}\right|[4,28]$ :

$$
h \Omega_{\mathrm{R}}=2 d\left|E_{\mathrm{vac}}\right| \sqrt{N V_{0}} .
$$

The product of $N V_{0}$ equals the number of oscillators that couple to the cavity mode, and $\left|E_{\mathrm{vac}}\right|$ is the amplitude of the vacuum electric field in the cavity with $v_{\mathrm{p}}=v_{\mathrm{TO}_{1}}=$ $0.95 \mathrm{THz}$. In our study, the parameters $N$ and $d$ were constant, and instead $\left|E_{\text {vac }}\right|$ and $V_{0}$ were changed by varying the gap size $G$ of the SRR.

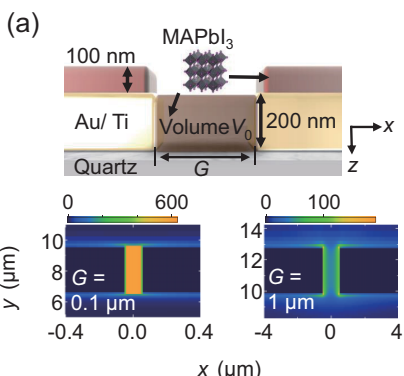

(b)
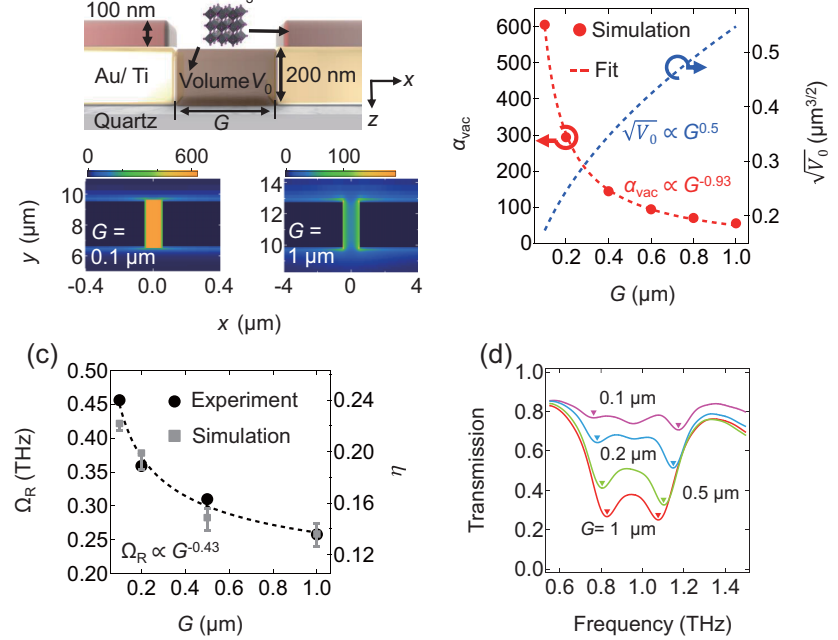

FIG. 3. (a) Upper panel: The region around the gap of the SRR model used in the FDTD simulations. The volume of $\mathrm{MAPbI}_{3}$ inside the gap is represented by $V_{0}$. Lower panel: The distribution of the enhancement factor $\alpha_{\mathrm{vac}}$ in the plane $z=-50 \mathrm{~nm}(z=0$ defines the quartz/SRR interface) for samples with $G=0.1$ and $1 \mu \mathrm{m}$. (b) Theoretical gap-size dependence of the enhancement factor $\alpha_{\text {vac }}$ averaged over $V_{0}$ (red circles) and its fitting result (red dashed curve). The blue dashed curve describes the gap-size dependence of the contribution of the oscillators in Eq. (5). (c) Experimental gap-size dependence of $\Omega_{\mathrm{R}}$ (black circles), the simulation result (gray squares), and the predicted curve proportional to $G^{-0.43}$ (black dashed curve). The error bars on gray squares refer to the deviation of simulation results when considering the thickness of $\mathrm{MAPbI}_{3}: t_{M A}\left(t_{\text {MAgap }}\right) \pm 20 \mathrm{~nm}$. The vertical axis on the right side of the figure represents the corresponding values of $\eta$. (d) Calculated transmission spectra of the on-resonance case. The polariton peaks are indicated by triangles.

Because $V_{0}$ linearly decreases as $G$ is decreased, the origin of the stronger coupling for narrower gaps [see Fig. 2(c)] is expected to be a relatively rapid increase in $\left|E_{\mathrm{vac}}\right|$. The increase in $\left|E_{\mathrm{vac}}\right|$ is denoted by $\alpha_{\mathrm{vac}}=\left|E_{\mathrm{vac}}\right| /\left|E_{\mathrm{vac} 0}\right|$, where $\left|E_{\mathrm{vac} 0}\right|$ is the amplitude of the vacuum electric field in free space without the cavity. $\alpha_{\mathrm{vac}}$ is an enhancement factor that depends on the gap size of the cavity. It can be assumed that the vacuum field is enhanced by the same amount as a real electric field in the gap of an SRR. Therefore, to quantitatively analyze the relationship between $\left|E_{\mathrm{vac}}\right|$ and $G$, we determined the enhancement factor of the vacuum field by using a finite-difference time-domain (FDTD) simulation. Here we calculated $\alpha_{\text {vac }}(\boldsymbol{r}, \omega)=|E(\boldsymbol{r}, \omega)| /\left|E_{0}(\boldsymbol{r}, \omega)\right|$, where $\left|E_{0}(\boldsymbol{r}, \omega)\right|$ and $|E(\boldsymbol{r}, \omega)|$ are the amplitude spectra of the real electric field in free space and around the SRR, respectively [43]. The geometry around the gap in the FDTD simulation is shown in Fig. 3(a) [32]. To calculate $\alpha_{\text {vac }}$, we considered the background refractive index of $\mathrm{MAPbI}_{3}(\approx 5.9)$. Representative calculation results for $\alpha_{\text {vac }}$ in the plane $50 \mathrm{~nm}$ above the quartz/Au interface at $v_{\mathrm{p}}=0.95 \mathrm{THz}$ are shown in the lower panels of Fig. 3(a). Figure 3(b) shows that the value of $\alpha_{\text {vac }}$ averaged over $V_{0}$ (red circles) is proportional to $G^{-0.93}$. Consequently, the relation between the vacuum field and the gap size is given by $\left|E_{\mathrm{vac}}\right|=\alpha_{\mathrm{vac}}\left|E_{\mathrm{vac} 0}\right| \propto G^{-0.93}$. The blue dashed curve in Fig. 3(b) plots $\sqrt{V_{0}} \propto G^{0.5}$, which describes 
(a)

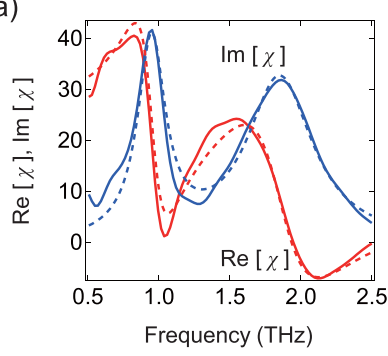

(b)

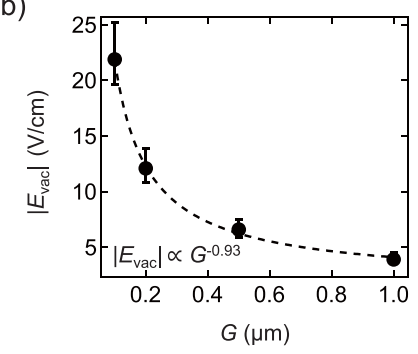

FIG. 4. (a) The susceptibility of $\mathrm{MAPbI}_{3}$ obtained from the transmission spectrum (solid curves) and the corresponding fitting result (dashed curves). Red and blue data represent the real and imaginary parts of the susceptibility, respectively. (b) The calculated gap-size dependence of the vacuum field in the gaps (black circles) together with the fitting result (black dashed curve). The calculated gap-size dependence of the vacuum field in the gaps (black circles) together with the fitting result (black dashed curve). The error bars come from the measurement error of thickness of $\mathrm{MAPbI}_{3}$ in gap $(200 \pm 20 \mathrm{~nm})$ and the fitting error of the parameter $\sqrt{N} d_{\mathrm{TO}_{1}}$ $\left[(2.8 \pm 0.2) \times 10^{-16} \mathrm{C} / \mathrm{m}^{0.5}\right]$.

the gap-size dependence of the contribution from the oscillators in Eq. (5). Therefore, $\Omega_{\mathrm{R}}$ should be proportional to $G^{-0.43}$ [the black dashed curve in Fig. 3(c)], which is in agreement with the values of $\Omega_{R}$ obtained in the experiments (the black circles). The origin of the gap-size dependence of the Rabi frequency can thus be summarized as follows. As the gap is narrowed, the vacuum electric field increases and the number of oscillators decreases. However, the increase in $\left|E_{\mathrm{vac}}\right|$ can more than compensate for the reduction in the number of oscillators, leading to an overall increase in $\Omega_{R}$ for narrower gaps. Figure 3(d) shows the theoretical spectra of the photonphonon polaritons calculated by the FDTD simulations. These spectra show the same behavior as the spectra in Fig. 2(b), and the simulated gap-size dependence of $\Omega_{\mathrm{R}}$ [gray squares in Fig. 3(c)] reproduces the experimental data well.

Equation (5) also allows us to estimate the vacuum field amplitude in the gap from the experimentally obtained Rabi frequency. For this estimation, the parameters $N$ and $d$ related to the TO phonons in the $\mathrm{MAPbI}_{3}$ thin film need to be determined. The linear susceptibility $\chi$ shown in Fig. 4(a) was determined from the complex transmission spectra $\tilde{T}(\omega)$ of the $850-$ nm-thick $\mathrm{MAPbI}_{3}$ film by THz-TDS. The microscopic expression of the susceptibility is given by [44]

$$
\begin{aligned}
\chi= & \chi_{\infty}+\frac{1}{3 \varepsilon_{0} \hbar} \sum_{i=\mathrm{TO}_{1}, \mathrm{TO}_{2}} \\
& \times\left[N d_{i}^{2}\left(\frac{1}{\omega_{i}-\omega-i \gamma_{i}}+\frac{1}{\omega_{i}+\omega+i \gamma_{i}}\right)\right],
\end{aligned}
$$

where the subscript $i$ refers to the phonon mode (we consider the $\mathrm{TO}_{1}$ and $\mathrm{TO}_{2}$ modes at 0.95 and $1.85 \mathrm{THz}$, respectively), $d_{i}$ is the dipole moment of each mode, $\omega_{i}$ is the angular frequency, and $\gamma_{i}$ is the linewidth. Note that the $\mathrm{TO}_{1}$ mode was coupled to the cavity in the experiment, and the $\mathrm{TO}_{2}$ mode affected the total susceptibility $\chi(\omega)$. The dashed curves in Fig. 4(a) are fits to Eq. (6), from which we obtain $\sqrt{N} d_{\mathrm{TO}_{1}} \approx(2.8 \pm 0.2) \times 10^{-16} \mathrm{C} / \mathrm{m}^{0.5}$. The parameter

$\sqrt{N} d_{\mathrm{TO}_{1}}$ describes the interaction strength between the $\mathrm{TO}_{1}$ phonons in $\mathrm{MAPbI}_{3}$ and light. A larger value of this parameter would correspond to a stronger absorption of light by the $\mathrm{TO}_{1}$ phonons. The value of $\sqrt{N} d_{\mathrm{TO}_{1}}$ of $\mathrm{MAPbI}_{3}$ is relatively small compared to the corresponding values of electronic excitations as shown in Refs. [34,45]. Therefore, it is more difficult to realize USC between the $\mathrm{TO}_{1}$ phonons and photons. In our work, we realized USC to phonons by using the strong electric field enhancement in SRRs with nanoscale gaps.

The amplitude of the vacuum field as a function of the gap size can be obtained by using Eq. (5). The result is shown in Fig. 4(b). When $G=0.1 \mu \mathrm{m}$, the estimated vacuum field in the $\mathrm{MAPbI}_{3}$ inside the gap is around $20 \mathrm{~V} / \mathrm{cm}$. We confirmed that this value has the same order of magnitude as the value calculated in Ref. [46]. Note that in Ref. [2], the vacuum field measured in free space is about $20 \mathrm{~V} / \mathrm{cm}$, but this value was derived using the different experimental conditions in the much higher frequency region of about $80 \mathrm{THz}$.

The reason for the enhancement of vacuum field can be understood in analogy to the enhancement of a real electric field in a metallic slit [47]: The electric field oscillating at $v_{\mathrm{p}}$ induces a current on the surface of the metal and leads to an accumulation of charge carriers around the gap, where the total charge $Q$ in the vicinity of one cavity wall does not depend on the gap size. The capacitive charging induces an electric near field in the gap. When $G$ is much smaller than the thickness of SRR $t_{\mathrm{Au}+\mathrm{Ti}}$, the charges are perfectly concentrated on the cavity walls, forming a parallel plate capacitor. However, in our case $G \sim t_{\mathrm{Au}+\mathrm{Ti}}$, and thus the charges spread away from the gap. Because the spreading distance is on the order of the gap size, the near field $E$ exhibits a strong gap-size dependence.

In conclusion, we have demonstrated that USC $(\eta \sim 0.24)$ between the SRR cavity photons and the $\mathrm{TO}_{1}$ phonon mode in $\mathrm{MAPbI}_{3}$ at $0.95 \mathrm{THz}$ can be achieved by narrowing the gap of the SRR to $0.1 \mu \mathrm{m}$. We have shown that, as the gap size becomes smaller, the contribution to $\eta$ from the vacuum field (which is proportional to $\alpha_{\mathrm{vac}}$ ) increases faster than that of the oscillators decreases, thus leading to an overall increase in $\eta$ for narrower gaps. On the basis of the experimentally obtained Rabi frequency and the linear susceptibility of $\mathrm{MAPbI}_{3}$ determined by THz-TDS, the average amplitude of the vacuum fluctuations inside the $0.1-\mu \mathrm{m}$-gap SRR was estimated to be $\sim 20 \mathrm{~V} / \mathrm{cm}$. Our results indicate that the photon-phonon coupling in the $\mathrm{THz}$ frequency region can reach a coupling deep in the USC regime if we confine the vacuum electric field on the nanometer scale, thereby providing us an alternative pathway to engineer vibration modes of materials that can significantly alter, for example, quantum properties in chemical reactions and phase transitions.

The authors acknowledge the financial support of a Grantin-Aid for Scientific Research (JP19H05465, JP21H01842) from the Japan Society for the Promotion of Science. The authors are grateful to Tian Li, Takahiro Moriyama, and Teruo Ono for their advice on the fabrication of the metal structures. 
[1] H. B. G. Casmir, On the attraction between two perfectly conducting plates, Proc. K. Ned. Akad. Wet. 51, 793 (1948).

[2] C. Riek, D. V Seletskiy, A. S. Moskalenko, J. F. Schmidt, P. Krauspe, S. Eckart, S. Eggert, G. Burkard, and A. Leitenstorfer, Direct sampling of electric-field vacuum fluctuations, Science 350, 420 (2015).

[3] I.-C. Benea-Chelmus, F. F. Settembrini, G. Scalari, and J. Faist, Electric field correlation measurements on the electromagnetic vacuum state, Nature 568, 202 (2019).

[4] M. Fox, Quantum Optics: An Introduction (Oxford University Press, Oxford, 2006).

[5] W. E. Lamb and R. C. Retherford, Fine structure of the hydrogen atom by a microwave method, Phys. Rev. 72, 241 (1947).

[6] F. J. Dyson, The electromagnetic shift of energy levels, Phys. Rev. 73, 617 (1948).

[7] S. K. Lamoreaux, Demonstration of the Casimir Force in the 0.6 to $6 \mu \mathrm{m}$ Range, Phys. Rev. Lett. 78, 5 (1997).

[8] F. Chen, U. Mohideen, G. L. Klimchitskaya, and V. M. Mostepanenko, Demonstration of the Lateral Casimir Force, Phys. Rev. Lett. 88, 101801 (2002).

[9] R. Stassi, A. Ridolfo, O. Di Stefano, M. J. Hartmann, and S. Savasta, Spontaneous Conversion from Virtual to Real Photons in the Ultrastrong-Coupling Regime, Phys. Rev. Lett. 110, 243601 (2013).

[10] S. De Liberato, C. Ciuti, and I. Carusotto, Quantum Vacuum Radiation Spectra from a Semiconductor Microcavity with a Time-Modulated Vacuum Rabi Frequency, Phys. Rev. Lett. 98, 103602 (2007).

[11] I. Carusotto, S. De Liberato, D. Gerace, and C. Ciuti, Back-reaction effects of quantum vacuum in cavity quantum electrodynamics, Phys. Rev. A 85, 023805 (2012).

[12] G. Romero, D. Ballester, Y. M. Wang, V. Scarani, and E. Solano, Ultrafast Quantum Gates in Circuit QED, Phys. Rev. Lett. 108, 120501 (2012).

[13] P. Nataf and C. Ciuti, Protected Quantum Computation with Multiple Resonators in Ultrastrong Coupling Circuit QED, Phys. Rev. Lett. 107, 190402 (2011).

[14] Y. Wang, J. Zhang, C. Wu, J. Q. You, and G. Romero, Holonomic quantum computation in the ultrastrong-coupling regime of circuit QED, Phys. Rev. A 94, 012328 (2016).

[15] J. A. Hutchison, T. Schwartz, C. Genet, E. Devaux, and T. W. Ebbesen, Modifying chemical landscapes by coupling to vacuum fields, Angew. Chem., Int. Ed. Engl. 51, 1592 (2012).

[16] A. Thomas, L. Lethuillier-Karl, K. Nagarajan, R. M. A. Vergauwe, J. George, T. Chervy, A. Shalabney, E. Devaux, C. Genet, J. Moran, and T. W. Ebbesen, Tilting a ground-state reactivity landscape by vibrational strong coupling, Science $\mathbf{3 6 3}$, 615 (2019).

[17] Y. Ashida, A. Imamoglu, J. Faist, D. Jaksch, A. Cavalleri, and E. Demler, Quantum Electrodynamic Control of Matter: Cavity-Enhanced Ferroelectric Phase Transition, Phys. Rev. X 10, 041027 (2020).

[18] S. Latini, D. Shin, S. A. Sato, C. Schäfer, U. De Giovannini, H. Hübener, and A. Rubio, The ferroelectric photo-groundstate of $\mathrm{SrTiO}_{3}$ : Cavity materials engineering, arXiv:2101.11313 [condmat, physics:physics].

[19] A. F. Kockum, A. Miranowicz, S. De Liberato, S. Savasta, and F. Nori, Ultrastrong coupling between light and matter, Nat. Rev. Phys. 1, 19 (2019).
[20] G. Günter, A. A. Anappara, J. Hees, A. Sell, G. Biasiol, L. Sorba, S. De Liberato, C. Ciuti, A. Tredicucci, A. Leitenstorfer, and R. Huber, Sub-cycle switch-on of ultrastrong light-matter interaction, Nature 458, 178 (2009).

[21] S. Gambino, M. Mazzeo, A. Genco, O. Di Stefano, S. Savasta, S. Patanè, D. Ballarini, F. Mangione, G. Lerario, D. Sanvitto, and G. Gigli, Exploring light-matter interaction phenomena under ultrastrong coupling regime, ACS Photon. 1, 1042 (2014).

[22] F. Todisco, M. De Giorgi, M. Esposito, L. De Marco, A. Zizzari, M. Bianco, L. Dominici, D. Ballarini, V. Arima, G. Gigli, and D. Sanvitto, Ultrastrong plasmon-exciton coupling by dynamic molecular aggregation, ACS Photon. 5, 143 (2018).

[23] J. Mornhinweg, M. Halbhuber, C. Ciuti, D. Bougeard, R. Huber, and C. Lange, Tailored Subcycle Nonlinearities of Ultrastrong Light-Matter Coupling, Phys. Rev. Lett. 126, 177404 (2021).

[24] D. J. Shelton, I. Brener, J. C. Ginn, M. B. Sinclair, D. W. Peters, K. R. Coffey, and G. D. Boreman, Strong coupling between nanoscale metamaterials and phonons, Nano Lett. 11, 2104 (2011).

[25] X. Jin, A. Cerea, G. C. Messina, A. Rovere, R. Piccoli, F. De Donato, F. Palazon, A. Perucchi, P. Di Pietro, R. Morandotti, S. Lupi, F. De Angelis, M. Prato, A. Toma, and L. Razzari, Reshaping the phonon energy landscape of nanocrystals inside a terahertz plasmonic nanocavity, Nat. Commun. 9, 763 (2018).

[26] R. Damari, O. Weinberg, D. Krotkov, N. Demina, K. Akulov, A. Golombek, T. Schwartz, and S. Fleischer, Strong coupling of collective intermolecular vibrations in organic materials at terahertz frequencies, Nat. Commun. 10, 3248 (2019).

[27] H. S. Kim, N. Y. Ha, J.-Y. Park, S. Lee, D.-S. Kim, and Y. H. Ahn, Phonon-polaritons in lead halide perovskite film hybridized with THz metamaterials, Nano Lett. 20, 6690 (2020).

[28] Q. Zhang, M. Lou, X. Li, J. L. Reno, W. Pan, J. D. Watson, M. J. Manfra, and J. Kono, Collective non-perturbative coupling of 2D electrons with high-quality-factor terahertz cavity photons, Nat. Phys. 12, 1005 (2016).

[29] W. Gao, X. Li, M. Bamba, and J. Kono, Continuous transition between weak and ultrastrong coupling through exceptional points in carbon nanotube microcavity exciton-polaritons, Nat. Photon. 12, 362 (2018).

[30] T. Handa, T. Yamada, M. Nagai, and Y. Kanemitsu, Phonon, thermal, and thermo-optical properties of halide perovskites, Phys. Chem. Chem. Phys. 22, 26069 (2020).

[31] F. Sekiguchi, H. Hirori, G. Yumoto, A. Shimazaki, T. Nakamura, A. Wakamiya, and Y. Kanemitsu, Enhancing the Hot-Phonon Bottleneck Effect in a Metal Halide Perovskite by Terahertz Phonon Excitation, Phys. Rev. Lett. 126, 077401 (2021).

[32] See Supplemental Material at http://link.aps.org/supplemental/ 10.1103/PhysRevResearch.3.L032021, which includes Refs. [25-28,33-35], for details of the sample preparation, the transmission spectra of all SRRs coated by $\mathrm{MAPbI}_{3}$ thin film, and the eigenvalue problem of the full Hopfield Hamiltonian.

[33] C. Maissen, G. Scalari, F. Valmorra, M. Beck, J. Faist, S. Cibella, R. Leoni, C. Reichl, C. Charpentier, and W. Wegscheider, Ultrastrong coupling in the near field of complementary split-ring resonators, Phys. Rev. B 90, 205309 (2014).

[34] G. Scalari, C. Maissen, D. Turčinková, D. Hagenmüller, S. De Liberato, C. Ciuti, C. Reichl, D. Schuh, W. Wegscheider, M. Beck, and J. Faist, Ultrastrong coupling of the cyclotron 
transition of a $2 \mathrm{D}$ electron gas to a $\mathrm{THz}$ metamaterial, Science 335, 1323 (2012).

[35] D. Hagenmüller, S. De Liberato, and C. Ciuti, Ultrastrong coupling between a cavity resonator and the cyclotron transition of a two-dimensional electron gas in the case of an integer filling factor, Phys. Rev. B 81, 235303 (2010).

[36] R. Ulbricht, E. Hendry, J. Shan, T. F. Heinz, and M. Bonn, Carrier dynamics in semiconductors studied with time-resolved terahertz spectroscopy, Rev. Mod. Phys. 83, 543 (2011).

[37] W. J. Padilla, A. J. Taylor, C. Highstrete, M. Lee, and R. D. Averitt, Dynamical Electric and Magnetic Metamaterial Response at Terahertz Frequencies, Phys. Rev. Lett. 96, 107401 (2006).

[38] Y. Mukai, H. Hirori, T. Yamamoto, H. Kageyama, and K. Tanaka, Nonlinear magnetization dynamics of antiferromagnetic spin resonance induced by intense terahertz magnetic field, New J. Phys. 18, 013045 (2016).

[39] N. Kim, S. In, D. Lee, J. Rhie, J. Jeong, D.-S. Kim, and N. Park, Colossal terahertz field enhancement using split-ring resonators with a sub-10 nm gap, ACS Photon. 5, 278 (2018).

[40] C. La-o-vorakiat, H. Xia, J. Kadro, T. Salim, D. Zhao, T. Ahmed, Y. M. Lam, J.-X. Zhu, R. A. Marcus, M.-E. MichelBeyerle, and E. E. M. Chia, Phonon mode transformation across the orthohombic-tetragonal phase transition in a lead iodide perovskite $\mathrm{CH}_{3} \mathrm{NH}_{3} \mathrm{PbI}_{3}$ : A terahertz time-domain spectroscopy approach, J. Phys. Chem. Lett. 7, 1 (2016).

[41] M. Sendner, P. K. Nayak, D. A. Egger, S. Beck, C. Müller, B. Epding, W. Kowalsky, L. Kronik, H. J. Snaith, A. Pucci, and
R. Lovrinčić, Optical phonons in methylammonium lead halide perovskites and implications for charge transport, Mater. Horiz. 3, 613 (2016).

[42] M. Nagai, T. Tomioka, M. Ashida, M. Hoyano, R. Akashi, Y. Yamada, T. Aharen, and Y. Kanemitsu, Longitudinal Optical Phonons Modified by Organic Molecular Cation Motions in Organic-Inorganic Hybrid Perovskites, Phys. Rev. Lett. 121, 145506 (2018).

[43] Y. Sanari, T. Tachizaki, Y. Saito, K. Makino, P. Fons, A. V. Kolobov, J. Tominaga, K. Tanaka, Y. Kanemitsu, M. Hase, and H. Hirori, Zener Tunneling Breakdown in Phase-Change Materials Revealed by Intense Terahertz Pulses, Phys. Rev. Lett. 121, 165702 (2018).

[44] R. W. Boyd and D. Prato, Nonlinear Optics, 3rd ed. (Academic, New York, 2008).

[45] M. Geiser, F. Castellano, G. Scalari, M. Beck, L. Nevou, and J. Faist, Ultrastrong Coupling Regime and Plasmon Polaritons in Parabolic Semiconductor Quantum Wells, Phys. Rev. Lett. 108, 106402 (2012).

[46] I.-C. Benea-Chelmus, Y. Salamin, F. F. Settembrini, Y. Fedoryshyn, W. Heni, D. L. Elder, L. R. Dalton, J. Leuthold, and J. Faist, Electro-optic interface for ultrasensitive intracavity electric field measurements at microwave and terahertz frequencies, Optica 7, 498 (2020).

[47] Y. Bahk, S. Han, J. Rhie, J. Park, H. Jeon, N. Park, and D. Kim, Ultimate terahertz field enhancement of single nanoslits, Phys. Rev. B 95, 075424 (2017). 